\title{
Deep bioinformatics expert system of analysis, modeling and interpretation of omics BigData of the human genome
}

\author{
A. Shlikht*, N. Kramorenko \\ Far Eastern Federal University, Vladivostok, Russia \\ *e-mail:schliht@mail.ru
}

Key words: genomics, proteomics, bigdata, bioinformatics, expert systems, data base

Motivation and Aim: An important task in omics BigData is to use an effective format and structure for data storage. The traditional sequential data file format does not provide sufficient performance for data access and subsequent automatic analysis, modeling, and interpretation. For this purpose, it is necessary to use the formats of databases and knowledge bases [1].

Methods and Algorithms: The transition from file sequential access to data and the use of procedural scripting programming languages to the format of databases and knowledge bases and declarative languages allows us to reach a qualitatively new level of artificial intelligence systems. For this purpose, it is necessary to restructure the primary file data of the world portals into local data stores with a database structure. Next, at the local level, a variety of methods of analysis, interpretation of databases and knowledge bases are used.

Results: The deep bioinformatics expert system has been developed, which allows to carry out work on the analysis, interpretation, modeling and diagnostics of omics BigData in an automatic mode at the local level with high efficiency. Depth of analysis, interpretation is provided by numerous associations between mutations, genes, transcripts, proteins (enzymes), reactions, metabolites, metabolic and signaling pathways, up to diseases. The system of associative relations allowed to build a deep subsystem of explanations, which is part of the expert system.

Conclusion: On the basis of the developed deep bioinformatics expert system, effective processes for the analysis, modeling, interpretation and diagnosis of omics BigData are carried out. The expert system can be useful for researchers, physicians and students.

\section{References}

1. Shlikht A.G., Kramorenko N.V. (2016) Bioinformatic expert system of analysis and interpretation of omics sequence of the human genome. MM-HPC-BBB-2016: P. 94. 